\title{
Geospatial (s)tools: integration of advanced epidemiological sampling and novel diagnostics
}

\author{
Giuseppe Cringoli', Laura Rinaldi', Marco Albonico², Robert Bergquist ${ }^{3}$, Jürg Utzinger ${ }^{4,5}$ \\ ${ }^{1}$ Unit of Parasitology and Parasitic Diseases, Department of Veterinary Medicine and Animal Productions, \\ University of Naples Federico II, Naples, Italy; ${ }^{2}$ Ivo de Carneri Foundation, Milan, Italy; ${ }^{3}$ Ingerod, Brastad, \\ Sweden; ${ }^{4}$ Department of Epidemiology and Public Health, Swiss Tropical and Public Health Institute, Basel, \\ Switzerland; ${ }^{5}$ University of Basel, Basel, Switzerland
}

\begin{abstract}
Large-scale control and progressive elimination of a wide variety of parasitic diseases is moving to the fore. Indeed, there is good pace and broad political commitment. Yet, there are some worrying signs ahead, particularly the anticipated declines in funding and coverage of key interventions, and the paucity of novel tools and strategies. Further and intensified research and development is thus urgently required. We discuss advances in epidemiological sampling, diagnostic tools and geospatial methodologies. We emphasise the need for integrating sound epidemiological designs (e.g. cluster-randomised sampling) with innovative diagnostic tools and strategies (e.g. Mini-FLOTAC for detection of parasitic elements and pooling of biological samples) and high-resolution geospatial tools. Recognising these challenges, standardisation of quality procedures, and innovating, validating and applying new tools and strategies will foster and sustain long-term control and eventual elimination of human and veterinary public health issues.
\end{abstract}

Keywords: diagnosis, disease control and elimination, epidemiology, geospatial tools, Mini-FLOTAC, parasitology, surveillance.

\section{Introduction}

Human and veterinary parasitology both present a number of challenges with regard to the necessary development of innovative tools that are required for an effective control and surveillance of a wide variety of diseases. What's more, bold objectives have been set for the elimination and eradication of malaria and a host of neglected tropical diseases (Alonso et al., 2011; WHO, 2012; Rollinson et al., 2013). Hence, there is a need to continue and intensify research and development for new and improved tools and strategies (e.g. drugs, vaccines and diagnostics) (Prichard et al., 2012; Alonso and Tanner, 2013). The link with geospatial methodologies provides support that moves activities forward (Utzinger et al., 2010; Chen et al., 2012). However, there is often a tendency to emphasise drug treatment and vaccines, while the importance of standardisation of geospatial strategies and diagnostics is neglected (Rinaldi et al., 2006; Bergquist et al., 2009; Solomon et al., 2012). It follows that further impetus

\footnotetext{
Corresponding author:

Giuseppe Cringoli

Unit of Parasitology and Parasitic Diseases

Department of Veterinary Medicine and Animal Productions

University of Naples Federico II

Via della Veterinaria 1, 80137 Naples, Italy

Tel. +39 081 253-6283; Fax +39081 253-6282

E-mail: cringoli@unina.it
}

for research on geospatial technology and links with more sensitive, standardised diagnostic techniques must take place.

Linking epidemiology, diagnosis and geospatial tools

\section{General considerations}

Modern epidemiological designs and innovative diagnostic tools applied at different spatial scales from territorial levels to single villages (farms) and at the unit of the host (human and animal) - are strongly needed for bridging scientific advances and public health in developing countries and the industrialised world alike. Global availability of geospatial health resource data and improved software analysis methodologies have enabled the development of digital "health maps" and transmission models for several parasitic infections of animals and humans (Brooker, 2007; Bergquist and Rinaldi, 2010; Soares Magalhães et al., 2011; Utzinger et al., 2011; Basáñez et al., 2012; Kelly et al., 2012; Malone and Bergquist, 2012; Pullan et al., 2012).

Representation of epidemiological data in the form of a map facilitates interpretation, synthesis and recognition of any changing frequency and pattern of infected cases and the appearance of clusters of parasitological phenomena. Moreover, maps are a convenient tool to foster discussion and dialogue among different 
stakeholders, particularly in connection with multicriteria decision analysis (Pfeiffer et al., 2008; Hongoh et al., 2011). However, when planning cross-sectional surveys, the use of spatial sampling strategies is neither often used for human infectious and parasitic diseases, nor in veterinary interventions. There is also a need for standardisation of multi-scale spatial sampling strategies based on the assumption that the sample (e.g. school, village or farm) is selected based on geographic location and local characteristics, such as climate, environment and management (an example is provided in Fig. 1). In the same sense, accurate diagnosis of parasitic infections is of pivotal importance for both individual patient management and population-based studies, such as drug efficacy trials and surveillance of parasitic disease control and elimination programmes, in both human and veterinary public health (Bergquist et al., 2009; Cringoli et al., 2010; Johansen et al., 2010). Moreover, the rigorous assessment of drug efficacy, monitoring community effectiveness of disease control interventions, verification of local elimination and early detection of resurgence depend strongly on the accuracy of diagnostic tools and sampling efforts (The malERA Consultative Group on Diagnoses and Diagnostics, 2011; McCarthy et al., 2012; Solomon et al., 2012).

Geospatial tools, including geographical information systems (GIS) and satellite-based technologies such as remote sensing and global positioning systems (GPS), coupled with geostatistical approaches, are increasingly and successfully applied at different levels from sam- pling to risk profiling of parasitic diseases (Rinaldi et al., 2006; Brooker, 2007; Simoonga et al., 2009; Machault et al., 2011; Utzinger et al., 2011). Indeed, this represents an innovative and useful way to communicate finding to field researchers and decision-makers and it is a powerful approach that also addresses the spatial targeting of parasite control, including the choice of treatment to be applied. The use of GIS and other geospatial tools, however, does by no means overcome the major concern of any empirical research, namely (parasitological) data quality (reviewed in Rinaldi et al., 2006). Therefore, achievement of high accuracy with regard to diagnosis of parasitic infections requires harmonisation of standardised protocols and multivalent techniques that are characterised by high sensitivity, specificity, precision, reproducibility and have the capacity to rapidly detect and monitor infections that pose human and veterinary public health problems (Cringoli et al., 2010; TDR Diagnostics Evaluation Expert Panel, 2010).

Implementation of standard protocols would lead to a more rigorous validation of the different diagnostic assays in use so that they can be employed with a better level of confidence at the different stages of control interventions (Bergquist et al., 2009). In addition, cost-effectiveness and sustainability are key issues on which standardised spatial sampling criteria (e.g. systematic grid sampling, proportional allocation, etc.) and diagnostic approaches should be based when conducting cross-sectional surveys in human and veterinary parasitology.

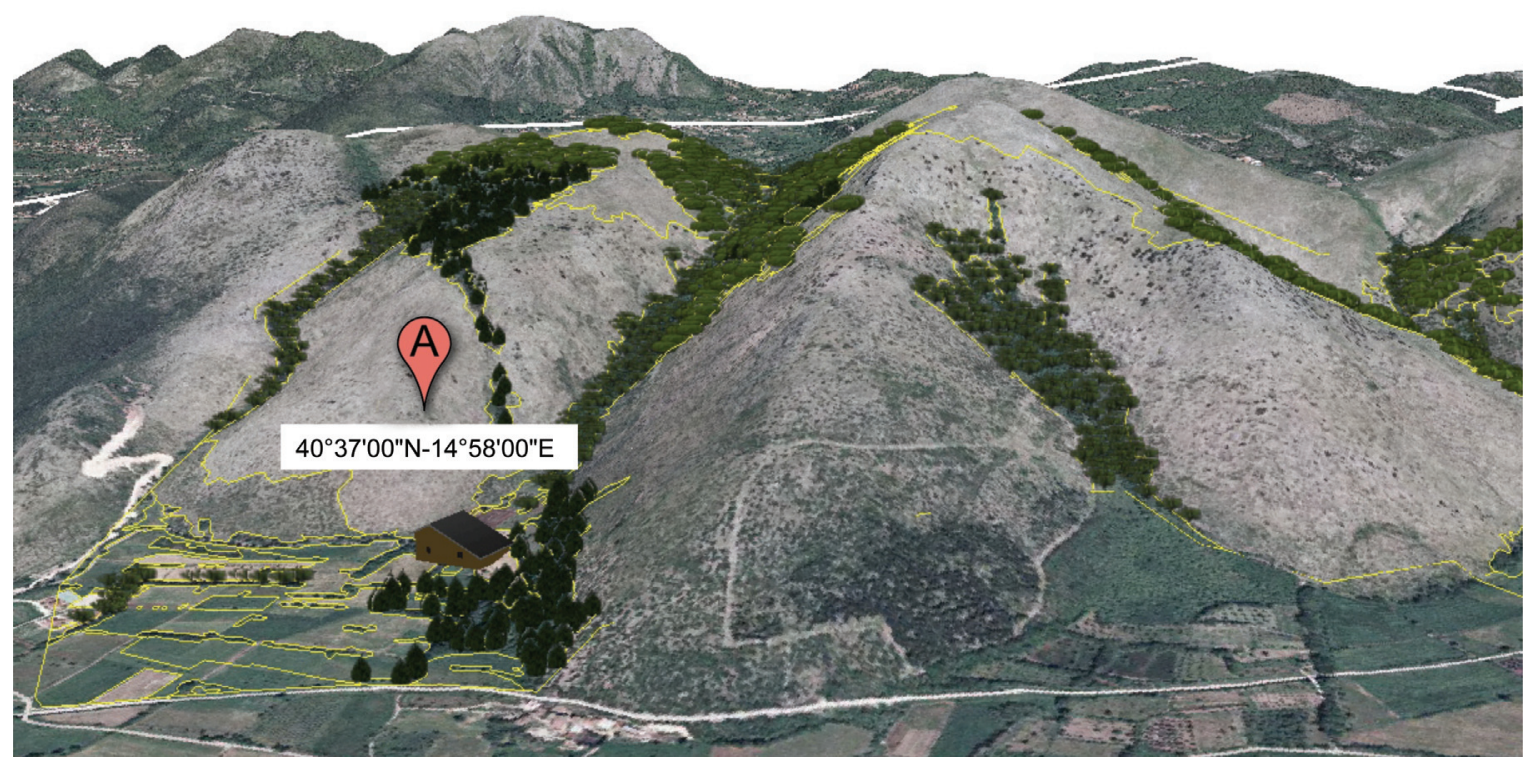

Fig. 1. An example of a geo-referenced sample (e.g. farm indicated by letter A) selected for a parasitological cross-sectional survey in base of geographic location and local characteristics (in yellow delimitation of pastures for remote sensing photo-interpretation). 


\section{Challenges and solutions ahead}

The international economic crisis and the resulting decline of research funds impose the need to resolve issues at considerably lower costs taking into account the logistical difficulties in conducting field surveys in human and veterinary parasitology. The standardisation and harmonisation of the use of innovative epidemiological and geospatial approaches (e.g. GIS, GPS and remote sensing) and novel diagnostic tools (e.g. the recently developed Mini-FLOTAC; see Cringoli et al. (2012); Fig. 2) for multivalent faecal egg counts (FECs) is advocated to standardise sustainable procedures and strategies for monitoring, surveillance and control of infections by parasitic organisms in animals and humans in the light of a geospatial-based "One Health" approach (Rinaldi et al., 2012).

The approach of sampling as recommended for human parasitology, especially for public health applications pertaining to helminthiasis, is usually based on choosing 5-10 schools in a target area and examining a random sample of 50 children from any of three upper classes (WHO, 2006). Cluster sampling (e.g. lot quality assurance sampling) or using sentinel surveillance sites are alternative approaches (Brooker et al., 2005; Steinmann et al., 2010; Belizario et al., 2013). These sampling strategies enable to extrapolate the magnitude and distribution of a given helminth infection within a circumcised geographical area, such as a district or an entire country. The World Health Organization recommends schools as sentinel sites, because the education system provides a readily accessible and convenient platform, and because schoolaged children are at highest risk of helminth infection. Schools should be chosen in a homogeneous agro-eco-

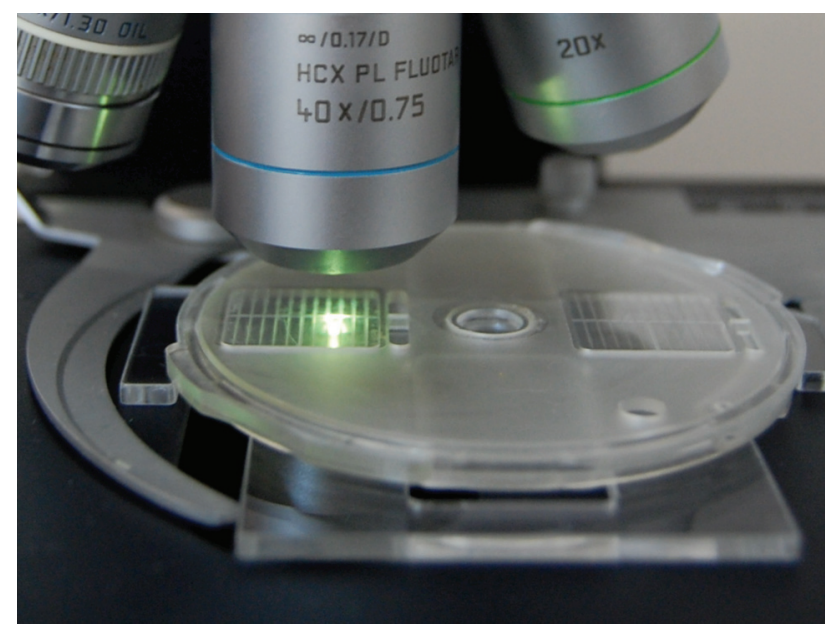

Fig. 2. Mini-FLOTAC under a light microscope. logical zone where transmission is more or less similar (WHO, 2006). The idea of ecological zone is not just based on climatic and environmental conditions but also driven by socio-economic parameters and population density. However, the criteria for identification and selection of homogeneous ecological zones are not clearly defined. Geospatial tools might help in defining them, and great progress has been made by designing open-access global databases for mapping, control and surveillance of helminthiases and other neglected tropical diseases, including web-based tools that show available data and matched remote sensing and geospatial information and model-based risk maps (Brooker et al., 2010; Hürlimann et al., 2011).

Geostatistical methods that take into account ecology and epidemiology of parasites and vectors/intermediate hosts have been discussed for malaria, soil-transmitted helminthiasis, schistosomiasis and other parasitic infections and proven to be an attractive model to predict parasite distribution and subsequently guide public health interventions (Brooker, 2007; Simoonga et al., 2009; Machault et al., 2011; Patil et al., 2011; Pullan et al., 2012). A promising approach to sampling inherited from veterinary parasitology is application of pooling of biological samples, such as blood, faeces and urine (Whittington et al., 2000; Mekonnen et al., 2013). Recently, such pooling approaches have been applied to fresh stool sample using the McMaster technique and to sodium acetate-acetic acid-formalin (SAF)-fixed faecal sample for the detection of intestinal parasites in man and results indicated that this is an efficient and potentially cost-effective strategy (Gaafar, 2011; Mekonnen et al., 2013). We applaud ongoing efforts to develop a mathematical framework that determines infection prevalence, which in turn can guide researchers and health decision makers to calculate sample size for egg reduction rate and lot quality assurance sampling. A web-based model for data entry calculating the prevalence, intensity and proportion of heavy intensity infections is under development.

\section{Mini-FLOTAC}

Recent studies pertaining to drug efficacy evaluation and detection of low-intensity intestinal parasite infections in animals and humans are pointing to the urge for low-cost, sensitive, accurate and easy-to-perform quantitative tests to be used in veterinary and public health (Knopp et al., 2008; Levecke et al., 2011). Mini-FLOTAC is a logical evolution of the FLOTAC technique (Cringoli et al., 2010), conceived in order to perform multivalent FECs for large-scale surveys in 


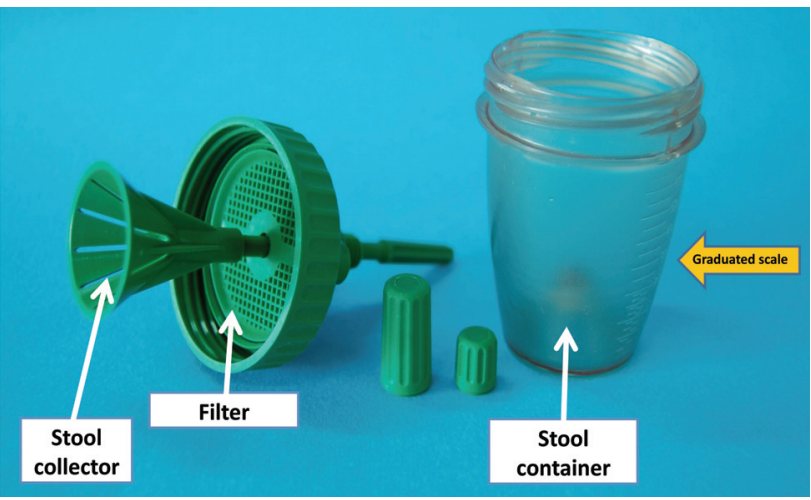

Fig. 3. Fill-FLOTAC and its main components.

laboratories with limited resources (i.e. where neither centrifugation nor other basic equipment are available). Mini-FLOTAC is particularly tailored for epidemiological monitoring and surveillance, where large numbers of faecal samples must be rapidly, yet reliably examined. Its user-friendly approach and high reproducibility rests on its simple design, which is based on only two components, the base and the reading disc. However, the device includes also two 1-ml flotation chambers designed for optimal examination of faecal sample suspensions (total volume $=2 \mathrm{ml}$ ). It is recommended that Mini-FLOTAC be used in combination with Fill-FLOTAC, a disposable sampling kit, which consists of a container, a collector and a filter (Fig. 3). Hence, Fill-FLOTAC facilitates the performance of the first four consecutive steps of the Mini-FLOTAC technique, i.e. sample collection and weighing, homogenisation, filtration and filling. The five steps of the MiniFLOTAC technique are depicted in Fig. 4.

Two operational advantages of the Mini-FLOTAC and Fill-FLOTAC in respect to currently more widely used diagnostic techniques, such as Kato-Katz and McMaster are that (i) it operates in a closed system and (ii) it can be performed on fixed faecal samples. Both

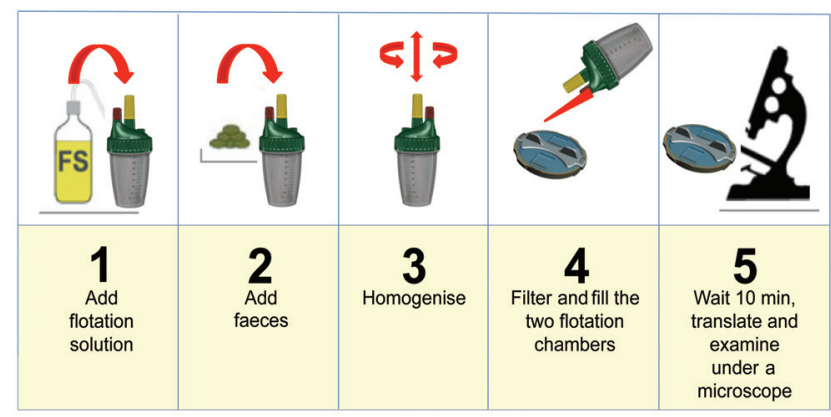

Fig. 4. The five consecutive steps of the Mini-FLOTAC technique. conditions allow, firstly, the protection of the operator from specific health hazards due to the manipulation of fresh stools samples and, secondly, offer an opportunity to processing samples not immediately after collection, but days or weeks after transfer to the laboratory. This is an important logistic advantage, which eases field work where laboratories are far away from collection sites (King et al., 2013), and also permits smooth performance of quality control.

Preliminary results show that Mini-FLOTAC is a promising technique for detecting and counting helminth eggs in animals and humans, and can be used in place of the FLOTAC technique (Cringoli et al., 2010), in laboratories where the centrifugation step cannot be performed. Mini-FLOTAC has been already validated in veterinary parasitology for the diagnosis of helminths (e.g. ascarids, hookworms, trichurids, gastro-intestinal nematodes and liver flukes) in pets and livestock (Cringoli, 2012; Cringoli et al., 2012). More recently, Mini-FLOTAC has been extended to human parasitology and broad-scale validation is underway for the diagnosis of major nematodes (e.g. soil-transmitted helminths) and trematodes (e.g. Schistosoma) parasitising man in different parts of the world. The results of this validation will be published in dedicated research papers to soon appear in the peer-reviewed international literature.

\section{Outlook}

We are confident that the use of geospatial tools, coupled with state-of-the-art epidemiological sampling and innovations in diagnostics (e.g. MiniFLOTAC) will help the advancement and standardisation of quality procedures for human and veterinary public health. The need for an accurate diagnosis, be it for rapid appraisal of high-risk areas, quantification of disease occurrence and burden, evaluation of control interventions, surveillance or verification of elimination, cannot be overemphasised.

Hence, standardised sampling and diagnostic procedures are required at different spatial levels, namely:

(i) at the local level: knowledge of the epidemiological scenario of parasites in a given territory;

(ii) at the national level: storage and analysis of parasitological information to facilitate local decisionmaking;

(iii) at the regional level: regional early warning, regional support and co-ordination; and

(iv) at the global level: risk modelling, trend monitoring and early warning systems. 
The future of geospatial "(s)tools" is already taking place now. A plethora of new technologies and webbased platforms are available for scientists, including virtual microscopy (Linder et al., 2008), virtual globes (Stensgaard et al., 2009) and vHealth (Bergquist and Tanner, 2012). These tools and technologies allow us to think differently a polyparasitic world!

\section{Acknowledgements}

The European Union 7th Framework Programme FP7-KBBE2011-5 under grant agreement n ${ }^{\circ} 288975$ (project GLOWORM) is acknowledged. The authors express sincere appreciation to Antonio Bosco, Giovanna Cappelli, Ida Guariglia, Davide Ianniello, Maria Paola Maurelli, Maria Elena Morgoglione, Paola Pepe, Mario Parrilla and Mirella Santaniello for their laboratory work for the Mini-FLOTAC validation.

\section{References}

Alonso PL, Brown G, Arevalo-Herrera M, Binka F, Chitnis C, Collins F, Doumbo OK, Greenwood B, Hall BF, Levine MM, Mendis K, Newman RD, Plowe CV, Rodríguez MH, Sinden R, Slutsker L, Tanner M, 2011. A research agenda to underpin malaria eradication. PLoS Med 8, e1000406.

Alonso PL, Tanner M, 2013. Public health challenges and prospects for malaria control and elimination. Nat Med 19, 150-155.

Basáñez MG, McCarthy JS, French MD, Yang GJ, Walker M, Gambhir M, Prichard RK, Churcher TS, 2012. A research agenda for helminth diseases of humans: modelling for control and elimination. PLoS Negl Trop Dis 6, e1548.

Belizario VY Jr, Totañes FI, de Leon WU, Ciro RN, Lumampao YF, 2013. Sentinel surveillance of soil-transmitted helminthiasis in preschool-aged and school-aged children in selected local government units in the Philippines: follow-up assessment. Asia Pac J Public Health (in press; doi: 10. 1177/1010539513483825).

Bergquist R, Johansen MV, Utzinger J, 2009. Diagnostic dilemmas in helminthology: what tools to use and when? Trends Parasitol 25, 151-156.

Bergquist R, Rinaldi L, 2010. Health research based on geospatial tools: a timely approach in a changing environment. J Helminthol 84, 1-11.

Bergquist R, Tanner M, 2012. Visual approaches for strengthening research, science communication and public health impact. Geospat Health 6, 155-156.

Brooker S, 2007. Spatial epidemiology of human schistosomiasis in Africa: risk models, transmission dynamics and control. Trans R Soc Trop Med Hyg 101, 1-8.

Brooker S, Hotez PJ, Bundy DAP, 2010. The global atlas of helminth infection: mapping the way forward in neglected tropical disease control. PLoS Negl Trop Dis 4, e779.

Brooker S, Kabatereine NB, Myatt M, Stothard JR, Fenwick A, 2005. Rapid assessment of Schistosoma mansoni: the validity, applicability and cost-effectiveness of the lot quality assurance sampling method in Uganda. Trop Med Int Health 10, 647-658. Chen JH, Wang H, Chen JX, Bergquist R, Tanner M, Utzinger J, Zhou XN, 2012. Frontiers of parasitology research in the People's Republic of China: infection, diagnosis, protection and surveillance. Parasit Vectors 5, 221.

Cringoli G, 2012. Copromicroscopic diagnosis of dicrocoeliosis: what's new? Proceedings of the XXVII Congress of the Italian Society of Parasitology (SOIPA), Alghero, 26-29 June 2012, p. 41. Cringoli G, Maurelli MP, Pepe P, Alfano S, Pennacchio S, Ianniello D, Rinaldi L, 2012. Mini-FLOTAC, a new tool for copromicroscopic diagnosis of Toxocara canis, Ancylostoma caninum and Trichuris vulpis in dogs. Proceedings of the XXVII Congress of the Italian Society of Parasitology (SOIPA), Alghero, 26-29 June 2012, p. 197.

Cringoli G, Rinaldi L, Maurelli MP, Utzinger J, 2010. FLOTAC: new multivalent techniques for qualitative and quantitative copromicroscopic diagnosis of parasites in animals and humans. Nat Protoc 5, 503-515.

Gaafar MR, 2011. Use of pooled sodium acetate acetic acid formalin-preserved fecal specimens for the detection of intestinal parasites. J Clin Lab Anal 25, 217-222.

Hongoh V, Hoen AG, Aenishaenslin C, Waaub JP, Bélanger D, Michel P, and The Lyme-MCDA Consortium, 2011. Spatially explicit multi-criteria decision analysis for managing vectorborne diseases. Int J Health Geogr 10, 70.

Hürlimann E, Schur N, Boutsika K, Stensgaard AS, Laserna de Himpsl M, Ziegelbauer K, Laizer N, Camenzind L, Di Pasquale A, Ekpo UF, Simoonga C, Mushinge G, Saarnak CFL, Utzinger J, Kristensen TK, Vounatsou P, 2011. Toward an open-access global database for mapping, control, and surveillance of neglected tropical diseases. PLoS Negl Trop Dis 5, e1404.

Johansen MV, Sithithaworn P, Bergquist R, Utzinger J, 2010. Towards improved diagnosis of zoonotic trematode infections in Southeast Asia. Adv Parasitol 73, 171-195.

Kelly GC, Tanner M, Vallely A, Clements A, 2012. Malaria elimination: moving forward with spatial decision support systems. Trends Parasitol 28, 297-304.

King JD, Endeshaw T, Escher E, Alemtaye G, Melaku S, Worku A, Adugna M, Melak B, Teferi T, Zerihun M, Gesese D, Tadesse Z, Mosher AW, Odermatt P, Utzinger J, Marti H, Ngondi J, Hopkins DR, Emerson PM, 2013. Intestinal parasite prevalence in an area of Ethiopia after implementing the SAFE strategy, enhanced outreach services, and health extension program. PLoS Negl Trop Dis 7, e2223.

Knopp S, Mgeni AF, Khamis IS, Steinmann P, Stothard JR, Rollinson D, Marti H, Utzinger J, 2008. Diagnosis of soiltransmitted helminths in the era of preventive chemotherapy: effect of multiple stool sampling and use of different diagnos- 
tic techniques. PLoS Negl Trop Dis 2, e331.

Levecke B, Speybroeck N, Dobson RJ, Vercruysse J, Charlier J, 2011. Novel insights in the fecal egg count reduction test for monitoring drug efficacy against soil-transmitted helminths in large-scale treatment programs. PLoS Negl Trop Dis 5, e1427. Linder E, Lundin M, Thors C, Lebbad M, Winiecka-Krusnell J, Helin H, Leiva B, Isola J, Lundin J, 2008. Web-based virtual microscopy for parasitology: a novel tool for education and quality assurance. PLoS Negl Trop Dis 2, e315.

Machault V, Vignolles C, Borchi F, Vounatsou P, Pages F, Briolant S, Lacaux JP, Rogier C, 2011. The use of remotely sensed environmental data in the study of malaria. Geospat Health 5, 151-168.

Malone JB, Bergquist R, 2012. Mapping and modelling neglected tropical diseases and poverty in Latin America and the Caribbean. Geospat Health 6, S1-5.

McCarthy JS, Lustigman S, Yang GJ, Barakat RM, García HH, Sripa B, Willingham AL, Prichard RK, Basáñez MG, 2012. A research agenda for helminth diseases of humans: diagnostics for control and elimination programmes. PLoS Negl Trop Dis 6, e1601.

Mekonnen Z, Meka S, Ayana M, Boger J, Vercruysse J, Levecke $\mathrm{B}, 2013$. Comparison of individual and pooled stool samples for the assessment of soil-transmitted helminth infection intensity and drug efficacy. PLoS Negl Trop Dis 7, e2189.

Patil AP, Gething PW, Piel FB, Hay SI, 2011. Bayesian geostatistics in health cartography: the perspective of malaria. Trends Parasitol 27, 246-253.

Pfeiffer C, Glaser S, Vencatesan J, Schliermann-Kraus E, Drescher A, Glaser R, 2008. Facilitating participatory multilevel decision-making by using interactive mental maps. Geospat Health 3, 103-112.

Prichard RK, Basáñez MG, Boatin BA, McCarthy JS, García HH, Yang GJ, Sripa B, Lustigman S, 2012. A research agenda for helminth diseases of humans: intervention for control and elimination. PLoS Negl Trop Dis 6, e1549.

Pullan RL, Sturrock HJW, Soares Magalhães RJ, Clements ACA, Brooker SJ, 2012. Spatial parasite ecology and epidemiology: a review of methods and applications. Parasitology 139, 1870-1887.

Rinaldi L, Gonzalez S, Guerrero J, Aguilera LC, Musella V, Genchi C, Cringoli G, 2012. A One-Health integrated approach to control fascioliasis in the Cajamarca valley of Peru. Geospat Health 6, S67-73.

Rinaldi L, Musella V, Biggeri A, Cringoli G, 2006. New insights into the application of geographical information systems and remote sensing in veterinary parasitology. Geospat Health 1 , 33-47.

Rollinson D, Knopp S, Levitz S, Stothard JR, Tchuem Tchuenté LA, Garba A, Mohammed KA, Schur N, Person B, Colley DG, Utzinger J, 2013. Time to set the agenda for schistosomiasis elimination. Acta Trop (in press; http://dx.doi.org/10.1016/ j.actatropica.2012.04.013).

Simoonga C, Utzinger J, Brooker S, Vounatsou P, Appleton CC, Stensgaard AS, Olsen A, Kristensen TK, 2009. Remote sensing, geographical information system and spatial analysis for schistosomiasis epidemiology and ecology in Africa. Parasitology 136, 1683-1693.

Soares Magalhães RJ, Clements ACA, Patil AP, Gething PW, Brooker S, 2011. The applications of model-based geostatistics in helminth epidemiology and control. Adv Parasitol 74, 267-296.

Solomon AW, Engels D, Bailey RL, Blake IM, Brooker S, Chen JX, Chen JH, Churcher TS, Drakeley CJ, Edwards T, Fenwick A, French M, Gabrielli AF, Grassly NC, Harding-Esch EM, Holland MJ, Koukounari A, Lammie PJ, Leslie J, Mabey DC, Rhajaoui M, Secor WE, Stothard JR, Wei H, Willingham AL, Zhou XN, Peeling RW, 2012. A diagnostics platform for the integrated mapping, monitoring, and surveillance of neglected tropical diseases: rationale and target product profiles. PLoS Negl Trop Dis 6, e1746.

Steinmann P, Usubalieva J, Imanalieva C, Minbaeva G, Stefiuk K, Jeandron A, Utzinger J, 2010. Rapid appraisal of human intestinal helminth infections among schoolchildren in Osh oblast, Kyrgyzstan. Acta Trop 116, 178-184.

Stensgaard AS, Saarnak CFL, Utzinger J, Vounatsou P, Simoonga C, Mushinge G, Rahbek C, Møhlenberg F, Kristensen TK, 2009. Virtual globes and geospatial health: the potential of new tools in the management and control of vector-borne diseases. Geospat Health 3, 127-141.

TDR Diagnostics Evaluation Expert Panel, 2010. Evaluation of diagnostic tests for infectious diseases: general principles. Nat Rev Microbiol 8 (12 Suppl), S17-29.

The malERA Consultative Group on Diagnoses and Diagnostics, 2011. A research agenda for malaria eradication: diagnoses and diagnostics. PLoS Med 8, e1000396.

Utzinger J, Bergquist R, Olveda R, Zhou XN, 2010. Important helminth infections in Southeast Asia: diversity, potential for control and prospects for elimination. Adv Parasitol 72, 1-30. Utzinger J, Rinaldi L, Malone JB, Krauth SJ, Kristensen TK, Cringoli G, Bergquist R, 2011. Geospatial health: the first five years. Geospat Health 6, 137-154.

Whittington RJ, Fell S, Walker D, McAllister S, Marsh I, Sergeant E, Taragel CA, Marshall DJ, Links IJ, 2000. Use of pooled fecal culture for sensitive and economic detection of Mycobacterium avium subsp. paratuberculosis infection in flocks of sheep. J Clin Microbiol 38, 2550-2556.

WHO, 2006. Preventive chemotherapy in human helminthiasis: coordinated use of anthelminthic drugs in control interventions: a manual for health professionals and programme managers. Geneva: World Health Organization.

WHO, 2012. Accelerating work to overcome the global impact of neglected tropical diseases - a roadmap for implementation. Geneva: World Health Organization. 